\title{
Molecular Weight Dependence of Primary Nucleation Rate of Polyethylene I. An Extended Chain Single Crystal
}

\author{
Mutsuo Nishi, Masamichi HikosaKa, ${ }^{\dagger}$ Swapan Kumer GHosh, \\ Akihiko Toda, and Koji Yamad** \\ Faculty of Integrated Arts and Sciences, Hiroshima University, \\ 1-7-1 Kagamiyama, Higashi-Hiroshima 739-8521, Japan \\ * Oita Research Center, Japan Polyolefins Co., Ltd., \\ 2 Nakanosu, Oita 870-0189, Japan
}

(Received January 22, 1999)

\begin{abstract}
Molecular weight $(M)$ dependence of the primary nucleation rate $(I)$ of an extended chain single crystal of polyethylene crystallized at high pressure $(P=0.4 \mathrm{GPa})$ was studied. We obtained for the first time an experimental formula that $I \propto M^{-1}$ which we named "power law of primary nucleation". We showed a well-known experimental formula that $I=I_{0} \exp \left(-\kappa / \Delta T^{2}\right)$, where $I_{0}$ is a constant proportional to the diffusion constant of molecules $(D), \kappa$ is related to the activation free energy for forming a critical nucleus $\left(\Delta G^{*}\right)$ and $\Delta T$ is the degree of supercooling. We showed that only $I_{0} \propto D$ decreases with increase in $M$ and $\kappa$ does not depend on $M$. From this results we concluded that $M$ dependence of $I$ is mainly controlled by the "chain sliding diffusion" process not by the formation process of a critical nucleus, that is, $I(M) \propto D(M) \propto P_{s}(M)$ and $\Delta G^{*}=$ const. Here $D$ can be regarded as an "expanded diffusion constant" and $P_{s}$ is a survival probability defined in "chain sliding diffusion" theory presented before by one of authors (MH). Based on these results we proposed a "chain sliding diffusion theory of primary nucleation" that the primary nucleation is a process of "chain sliding diffusion" within the nucleus which requires disentanglement of molecular chains within the interface between the nucleus and the melt. The theory explained well the observed power law.

KEY WORDS Nucleation / Nucleation Rate / Molecular Weight / Polyethylene / Power Law / Sliding Diffusion /
\end{abstract}

Primary nucleation is important onset stage ${ }^{1}$ in crystallization of materials from an isotropic phase, such as the melt, the solution, gas and amorphous solid or from other phases, such as liquid crystalline one. The primary nucleation mechanism of atomic or low molecular weight systems has been studied well, ${ }^{2-5}$ while that of polymers has not been studied well and has been left unsolved for long years. ${ }^{6-10}$

It is obvious that the molecular weight $(M)$ is one of the most important parameters of poymer systems and it has been well known that $M$ strongly affects the nucleation process of polymers, which strongly controls the crystallization behaviors. But the $M$ dependence of polymer nucleation has not been studied well as far as authors' knowledge, therefore the mechanism of it has not been solved at all, although it is very interesting and important for both polymer science and technology to solve it.

The main purpose of this work is to show a reliable experimental formula of $M$ dependence of the primary nucleation rate $(I)$ of polyethylene $(\mathrm{PE})$ for the first time and to propose a new mechanism of the $M$ dependence of the primary nucleation which we will name "chain sliding diffusion theory of primary nucleation." The theory will introduce the topological nature of chain molecules to the nucleation mechanism of polymer systems.

As to the $M$ dependence of the lateral growth $\operatorname{rate}(V)$, we have already shown in the previous papers ${ }^{1,12}$ that $V \propto M^{-H}$ where $H$ is a constant (Figure 1). We named it "power law of the lateral growth." $H$ depends on morphologies of folded chain and extended chain crystals

\footnotetext{
† To whom correspondence should be addressed.
}

(FCC and ECC), that is $H=1.8$ and 0.7 for FCC and for ECC, respectively. From this result, it is concluded that chain sliding diffusion on the interface between the melt and crystal controls the $M$ dependence of the $V$ (Figure 2). Another purpose of this work is to make clear if the "power law" can be also found in $M$ dependence of nucleation rate or not.

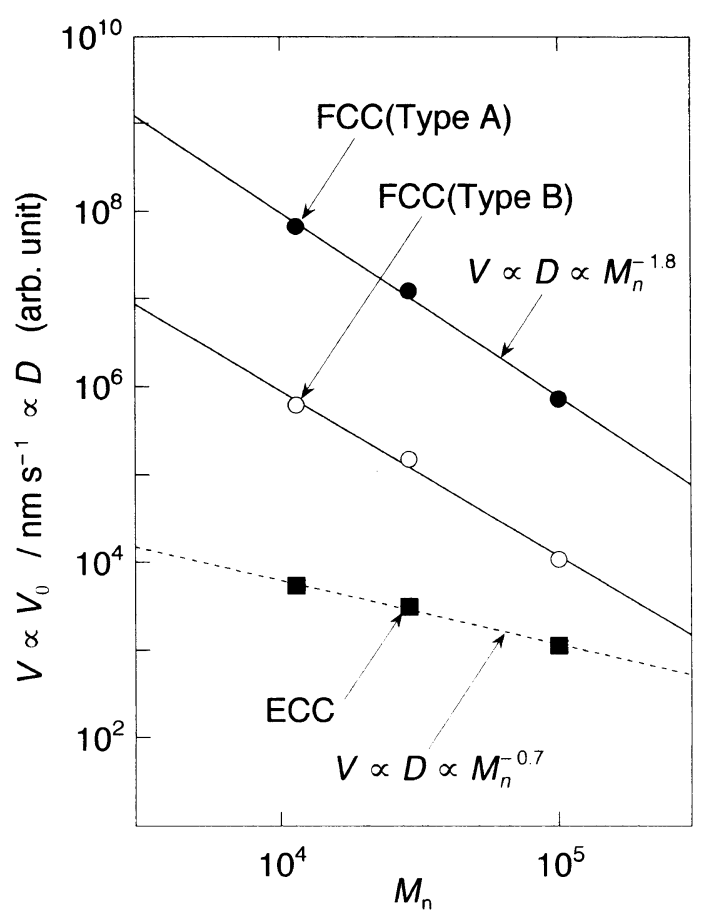

Figure 1. Molecular weight $(M)$ dependence of the lateral growth rate $(V)$ of PE single cyrstals, indicating $V \propto M^{-H}$ where $H$ is a constant. This was named "power law of the lateral growth." 11,12 


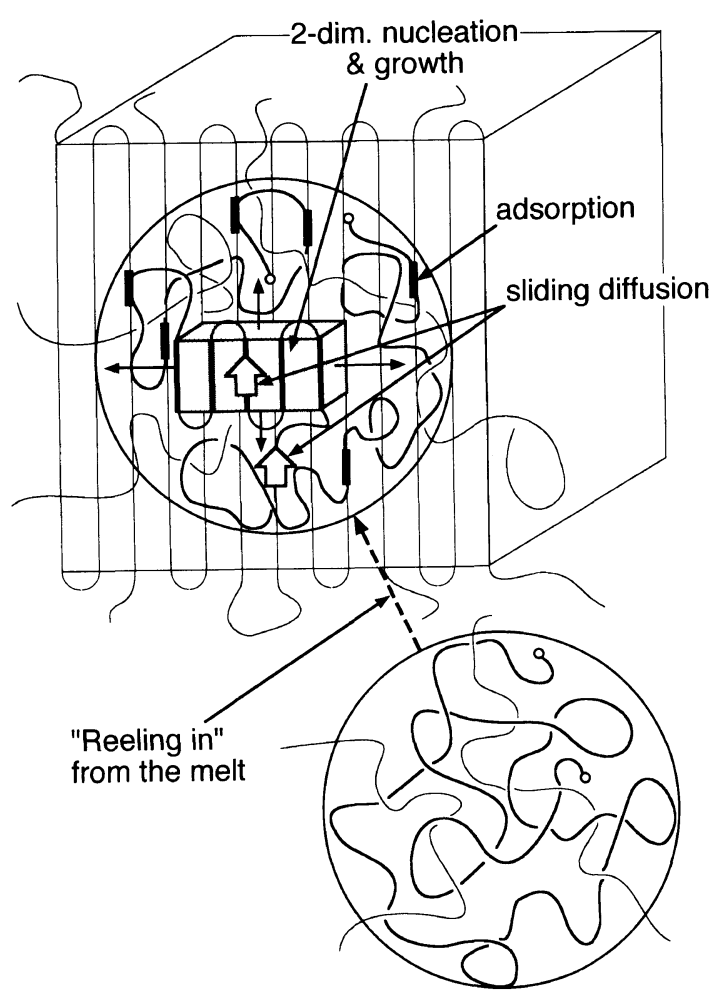

Figure 2. Schematic illustration of the sliding diffusion model of two dimensional nucleation on the surface of a single cyrstal.

It should be noted that there is a strong contrast in the crystallization process between atomic or low molecular weight systems and polymer systems due to "topological nature" of polymer chains as shown below. Figure 3 illustrates this contrast schematically. In the former system the nucleation is a rather simple process where each atom or molecule can be transported and rearranged independently from the isotropic phase into crystalline lattice points (Figure 3a).

In the latter system, the nucleation is very complicated process, because each polymer should be transported and rearranged under the strong restriction that the molecular chain must not be "cut."* The restriction can be described precisely by introducing a topological concept that one dimensional topological nature should be invariant during crystallization. Therefore the restriction can be called topological one. In this case, it is natural that the chain has to slide along the chain axes for rearrangement of chains from the melt into crystalline lattice, i.e., in the nucleation and growth processes. (Figure 3b). ${ }^{13}$

The topological nature within the melt was well studied by De Gennes ${ }^{14}$ and Doi and Edwards. ${ }^{15}$ The topological nature in polymer nucleation has not been studied yet. It is expected that the topological restriction increases with increase in $M$. A study of $M$ dependence of crystallization behavior is important to clarify the crystallization mechanism of polymer systems.

It is the basic concept in classical nucleation theory that in the onset of nucleation process, small nuclei (which is sometimes named "embryo" when its size is smaller than that of a critical nucleus) are very frequently generated and diminished within the isotropic phase by thermal fluctuation under supercooled or supersaturated (a) Atomic system

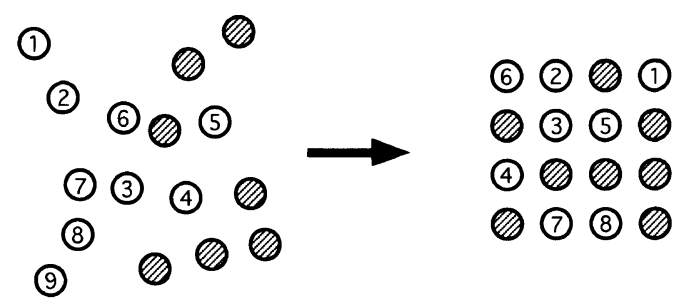

Isotropic phase $\longrightarrow$ Crystalline phase

\section{(b) Polymer system}

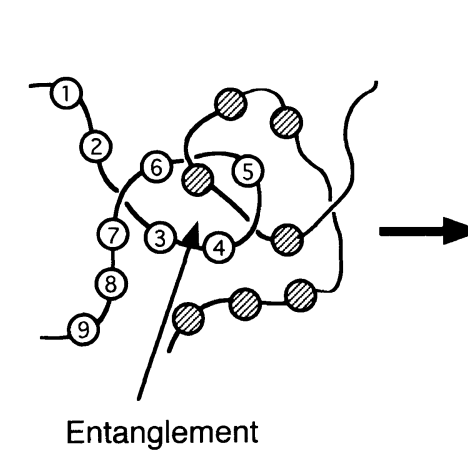

(Topological restriction)
Figure 3. Difference in crystallization behavior between atomic or short chain molecular system and polymer one. (a) Atoms or short chain molecules can be independently rearranged on each lattice point, while (b) the order of the repeating units within a polymer chain should be kept to be the same during rearranging process. Therefore, a chain should slide along its chain axis and disentangle for rearranging onto the lattice points.

conditions. ${ }^{4}$ The frequency roughly estimated by well known frequency factor $(k T / h)$ is as large as $10^{13}$, where $k$ is Boltzmann constant and $T$ is temperature.

Classical nucleation theories given by Becker and Döring ${ }^{16}$ and Turnbull and Fisher ${ }^{2}$ showed that nucleation rate $(I)$ is a product of probability of diffusion of atoms or molecules and that of formation of a critical nucleus. There are three kinds of diffusions, within the isotropic phase, the interface (between the isotropic and the crystalline phases) and the crystalline phase.

In the case of polymer system, topological nature of polymer chains takes an important role in the above three kind of diffusions. It should be emphasized that the diffusion within the crystalline phase is especially important in the case of polymer nucleus, which has been shown for the first time in the "chain sliding diffusion" theory proposed by Hikosaka. ${ }^{17,18} \mathrm{He}$ succeeded to explain the origin of two typical morphologies of polymer crystals, i.e., $\mathrm{FCC}^{19}$ and ECC. ${ }^{20}$ It is obvious that any nucleus, whether a primary nucleus or a two-dimensional nucleus, and a crystal can not grow or thicken without the "chain sliding diffusion" within them.

We will define here much more clearly that the "chain sliding diffusion" is "self-diffusion of a polymer chain molecule along its chain axis in some anisotropic

* In this study it is assumed that no chemical reaction such as cutting of bond occurs during the crystallization process. 
potential field as seen within a nucleus, a crystal or the interface between the crystalline and the isotropic phases." Frank and Tosi suggested that "the crystal is likely to change after growth by creeping displacements of the molecular chains tending to even out the segment lengths," 21 which can be regarded as a primitive suggestion of the "chain sliding diffusion".

We have shown in the previous paper ${ }^{11,12}$ that the sliding diffusion within the interface between the crystal and the melt controls $M$ dependence of $V$ of PE single crystals.

It is natural to consider that the topological effect on nucleation increases with increase in $M$ as shown in the $M$ dependence of $V \cdot{ }^{11,12} M$ is one of the most important parameters in determining structures and physical properties of polymers which reflects the topological nature of polymer chain molecules. $M$ dependence of nucleation behavior will give important information to solve the nucleation mechanism of polymers.

Nucleation rate $(I)$ is defined by increase of number density $(v)$ of nuclei within the melt per unit time $(t),{ }^{10}$ that is,

$$
I \equiv \frac{\mathrm{d} v}{\mathrm{~d} t}
$$

Becker and Döring ${ }^{16}$ and Turnbull and Fisher ${ }^{2}$ showed that $I$ is expressed by

$$
I=I_{0} \exp \left(-\frac{\Delta G^{*}}{k T}\right)
$$

where $I_{0}$ is a constant in proportion to self-diffusion constant $(D), \Delta G^{*}$ is free energy for forming a critical nucleus and $k T$ is thermal energy. ${ }^{4,5} I_{0}$ and $D$ are usually expressed by

$$
I_{0} \propto D \propto \exp \left(-\frac{\Delta E}{k T}\right)
$$

where $\Delta E$ is an activation energy for self-diffusion of a unit, such as an atom or a repeating unit of polymer. Frank and Tosi formulated $I$ considering net flow of a sequential process ${ }^{21}$ which represented all nucleation process from the onset of growth of nucleus into macroscopic nucleus. They proposed a basic formula for $I$ (eq 13 in ref 21 ) and applied it to so called "secondary nucleation" model proposed by Lauritzen and Hoffman. ${ }^{6}$ Hikosaka expanded the Frank-Tosi's theory and proposed the "chain sliding diffusion" theory. ${ }^{17,18}$ The theory gave $I$,

$$
I \propto \exp \left(-\frac{\Delta E^{*}}{k T}\right) P_{\mathrm{s}} \exp \left(-\frac{\Delta G^{*}}{k T}\right)
$$

where $\Delta E^{*}$ is a $\Delta E$ for the stage of a critical nucleation and $P_{s}$ is named "survival probability." $P_{s}$ is defined by

$$
P_{\mathrm{s}} \equiv \frac{1}{\sum_{m=0}^{\infty} \exp \left[\left(\frac{\Delta G_{m}}{k T}+\frac{\Delta E_{m}}{k T}\right)-\left(\frac{\Delta G^{*}}{k T}+\frac{\Delta E^{*}}{k T}\right)\right]}
$$

where $m$ is a stage number in the sequential process, $\Delta G_{m}$ is the free energy for forming a nucleus of the $m$ th stage, $\Delta E_{m}$ is a $\Delta E$ from the $m$ th to the $(m+1)$ th stage. Here we omit the term of glass transition temperature $\left(T_{g}\right)$, because it is not serious in this study. In the theory $\Delta E_{m}$ contains an effect of the "chain sliding diffusion" and $\Delta E_{m}$ is a function of lamellar thickness at the $m$ th stage $\left(l_{m}\right)$. Thus we have a corresponding relation by comparing eq 2,3 , and 4 .

$$
I_{0} \propto D \propto \exp \left(-\frac{\Delta E^{*}}{k T}\right) P_{\mathrm{s}}
$$

Therefore we will name $D$ "expanded diffusion constant" expanded to a topological linear chain systems. In this meaning we will call the diffusion process "expanded diffusion process." Thus we have

$$
I \propto D \exp \left(-\frac{\Delta G^{*}}{k T}\right)
$$

It is well known that

$$
\Delta G^{*}=\frac{C}{\Delta T^{2}}
$$

where $C$ is a constant related to surface free energies and enthalpy of fusion and $\Delta T$ is degree of supercooling. ${ }^{17,18}$ Substitution of eq 8 into eq 2 leads,

$$
I=I_{0} \exp \left(-\frac{\kappa}{\Delta T^{2}}\right)
$$

where $\kappa$ is defined by

$$
\kappa \equiv \frac{C}{k T}
$$

The first purpose of this study is to obtain a reliable experimental formula for $M$ dependence $I$ of $\mathrm{PE}$ and to make clear that if the "power law' can be universally found on $M$ dependence of nucleation rate or not. It will be shown that $I \propto M^{-1}$ for the first time, which will be named "power law of primary nucleation." The second purpose is to solve the problem what controls the $M$ dependence of $I$. It will be shown that the "expanded self-diffusion process" of chain molecules controls the $M$ dependence of $I$, while the critical nucleation process does not. The final purpose is to propose a "chain sliding diffusion theory of polymer nucleation" based on these results. It will be shown finally that the primary nucleation process is dominated by the "chain sliding diffusion" within the nucleus which requires disentanglement of chain molecules within the interface between the nucleus and the melt.

\section{EXPERIMENTAL}

Four kinds of well fractionated PE (named $13 \mathrm{~K}, 30 \mathrm{~K}$, $99 \mathrm{~K}$, and $256 \mathrm{~K}$, respectively) supplied by Japan Polyolefins Co., Ltd were used in this study. (Table I). Here $13 \mathrm{~K}, 30 \mathrm{~K}$, etc. indicate $M_{n}=13 \times 10^{3}, 30 \times 10^{3}$, etc., respectively. $M_{n}$ and $M_{w}$ are number averaged and weight averaged molecular weight, respectively. All samples were well fractionated from the same PE named "mother PE" which was polymerized with metallocene catalyst system. The mother PE was crystallized from the solution onto a surface of supports (Celite545) by slow cooling $\left(10^{\circ} \mathrm{Ch}^{-1}\right)$ process, where mixed solvent of xylene (good solvent) and ethylene glycol mono-ethyl 
Table I. $M_{n}, M_{w}$ and molecular weight distribution of samples

\begin{tabular}{lccc}
\hline Sample name & $M_{n}$ & $M_{w}$ & $M_{w} / M_{n}$ \\
\hline Mother PE & $47.8 \times 10^{3}$ & $116 \times 10^{3}$ & 2.42 \\
J-PE1 $(13 \mathrm{~K})$ & $12.9 \times 10^{3}$ & $16.5 \times 10^{3}$ & 1.28 \\
J-PE2 $(30 \mathrm{~K})$ & $29.5 \times 10^{3}$ & $34.0 \times 10^{3}$ & 1.15 \\
J-PE6 $(99 \mathrm{~K})$ & $99.4 \times 10^{3}$ & $114 \times 10^{3}$ & 1.14 \\
J-PE8 $(256 \mathrm{~K})$ & $256 \times 10^{3}$ & $314 \times 10^{3}$ & 1.24 \\
\hline
\end{tabular}

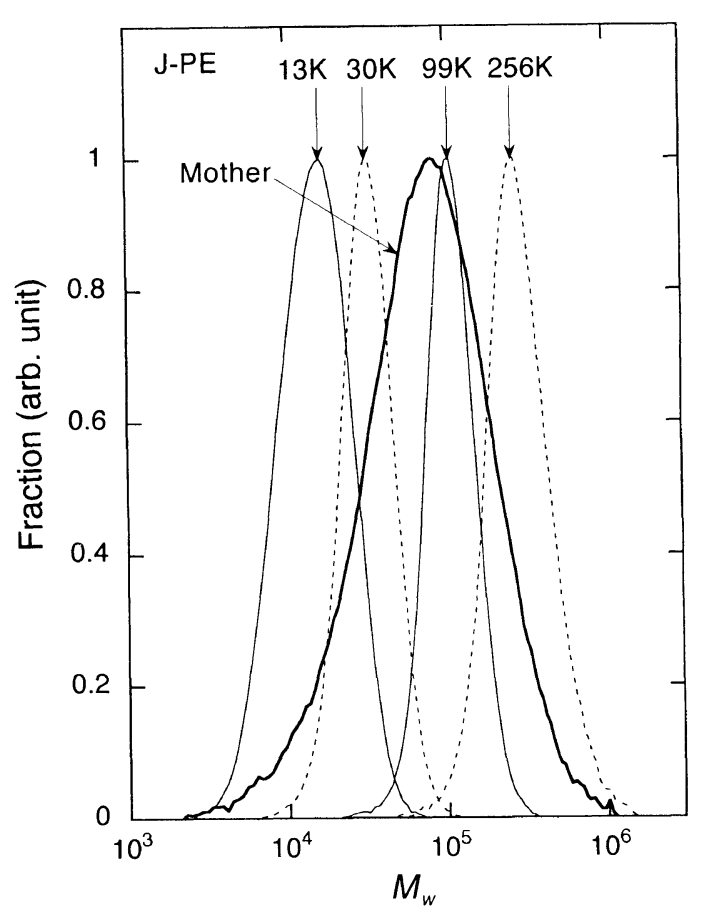

Figure 4. GPC profiles of fractionated samples, $13 \mathrm{~K}, 30 \mathrm{~K}, 99 \mathrm{~K}$, and $256 \mathrm{~K}$ and "mother PE".

ether (poor one) were used. Fractionation was carried out by column elution at $127^{\circ} \mathrm{C}$. The sample was dissolved in the mixed solvent. The ratio of good solvent was increased step by step from $0 \%$ to $64 \%$. PE was deposited from the fractionated solution at room temperature and it was recovered by filtration. Molecular weight distribution of each fraction was measured by gel permeation chromatography (GPC). Figure 4 shows the GPC profiles in each samples. $M_{n}, M_{w}$ and molecular weight distribution of the samples are shown in Table I. Number of branches within the mother PE measured by means of ${ }^{13} \mathrm{C}$ nuclear magnetic resonance $\left({ }^{13} \mathrm{C} \mathrm{NMR}\right)$ was small as shown in Table II.

Isothermal crystallization of these samples was isothermally carried out under high pressure from the melt into the hexagonal phase. The pressure $(P)$ was $0.4 \mathrm{GPa}$. The range of $\Delta T$ was $3.3-9.4^{\circ} \mathrm{C}$. We defined $\Delta T$ using equilibrium melting temperature $\left(T_{m}\right)$ estimated by Wunderlich's method. ${ }^{22}$ We counted a number of crystals observed within a visual field of polarizing optical microscope. The number of single crystals should correspond to that of nucleus. Therefore number density of nucleus $v$ was estimated from the number of crystals within a unit volume of the melt. The details of experimental procedure were given in ref 23 and 24 .
Table II. Number of branches in mother polyethylene

\begin{tabular}{lc} 
Type of branch & $\begin{array}{c}\text { Number of branches } \\
(1 / 1000 \mathrm{C})\end{array}$ \\
\hline$-\mathrm{CH}_{3}$ branch & 0.2 \\
$-\mathrm{CH}_{2} \mathrm{CH}_{3}$ branch & 0.2 \\
Higher branch & 0.1 \\
\hline
\end{tabular}

(a) $13 \mathrm{~K}$

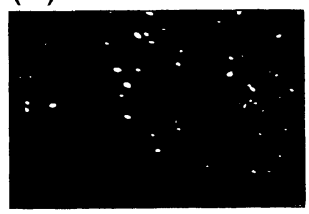

(b) $30 \mathrm{~K}$

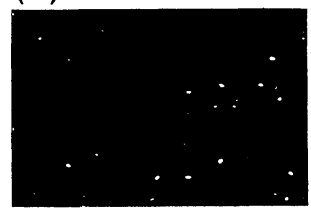

(c) $99 \mathrm{~K}$

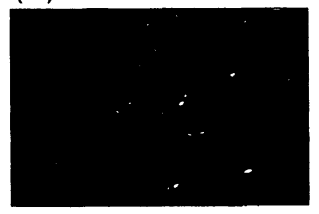

(d) $256 \mathrm{~K}$

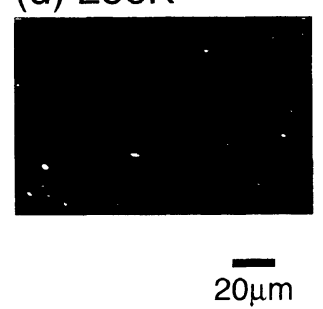

Figure 5. Typical polarizing optical micrographs of growing ECSCs. $\Delta T=4.2 \mathrm{C}, P=0.4 \mathrm{GPa}$; (a) $13 \mathrm{~K}$; (b) $30 \mathrm{~K}$; (c) $99 \mathrm{~K}$; (d) $256 \mathrm{~K}$. Scale bar shows $20 \mu \mathrm{m}$.

\section{RESULTS}

\section{Morphology}

Figures 5(a)-(d) show polarizing optical micrographs of crystals in samples $13 \mathrm{~K}, 30 \mathrm{~K}, 99 \mathrm{~K}$, and $256 \mathrm{~K}$, respectively. Isolated cigar-like crystals were observed in all $M_{n}$. The morphology is the same as that of extended chain single crystals (ECSCs) reported in our previous studies, ${ }^{11,25}$ which indicates that an isolated ECSC was formed in all $M_{n}$. One isolated single crystal should be generated from one primary nucleus. Therefore we can regard the number of crystals as that of nuclei in this study.

\section{Nucleation Rate}

Steady Nucleation Process. Typical number density of nucleus $v$ observed for each sample, $13 \mathrm{~K}, 30 \mathrm{~K}, 99 \mathrm{~K}$, and $100 \mathrm{~K}$, is plotted against crystallization time $(t)$ for $\Delta T=4.1^{\circ} \mathrm{C}$ in Figure 6. It is clearly shown that $v$ increased linearly with increase in $t$ for all $M_{n}$. This shows that this nucleation process is steady one. Nucleation rate $I$ was obtained from the slope of the lines. It is to be noted that $I$ decreased with increase in $M_{n}$ at a $\Delta T$.

$\Delta T$ Dependence of Nucleation Rate, $I . \quad \log I$ is plotted against $\Delta T^{-2}$ for samples, $13 \mathrm{~K}, 30 \mathrm{~K}, 99 \mathrm{~K}$, and $256 \mathrm{~K}$, in Figure 7. $\log I$ decreased lineally with increase in $\Delta T^{-2}$ in all $M_{n}$, from which we obtained well known experimental formula of $\Delta T$ dependence of $I$ given by eq 9 , $I=I_{0} \exp \left(-\kappa / \Delta T^{2}\right)$. This confirmed that this process is controlled by three-dimensional nucleation process. $I_{0}$ can be obtained from an intercept of the vertical axis and $\kappa$ from the slope of a line in Figure 8.

$I_{0}$ given by eq 6 is a function of $T$ in general, but $I_{0}$ can be approximated to be constant in this study. Because 


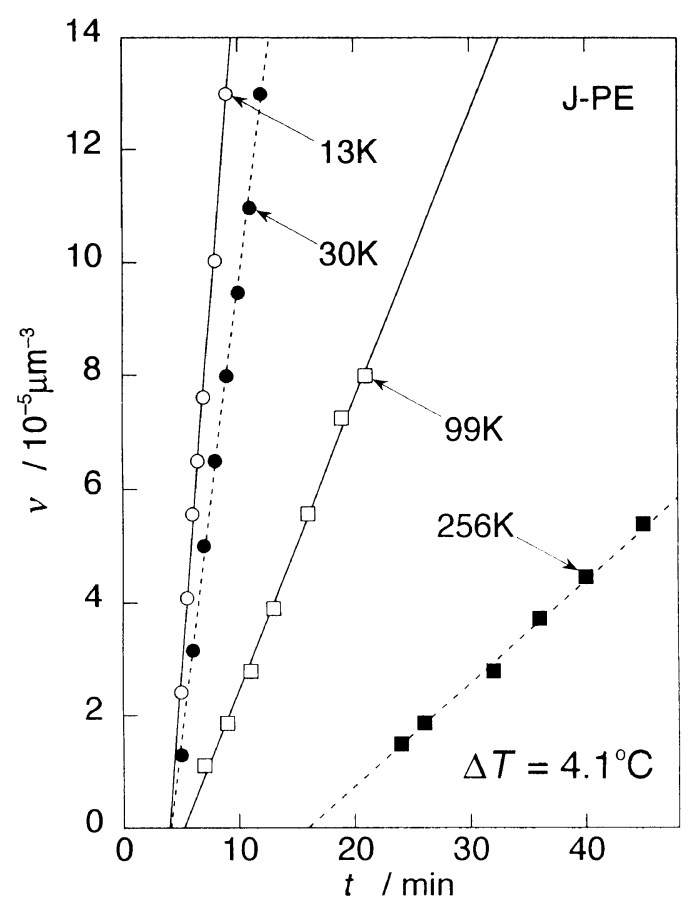

Figure 6. Plot of $v$ against $t$ in $M_{n}=13 \mathrm{~K}, 30 \mathrm{~K}, 99 \mathrm{~K}$, and $256 \mathrm{~K}$ at $\Delta T=4.1^{\circ} \mathrm{C}$. The lines show the best fit of the plots. The slope corresponding to the $I$ decreased with increase of $M$.

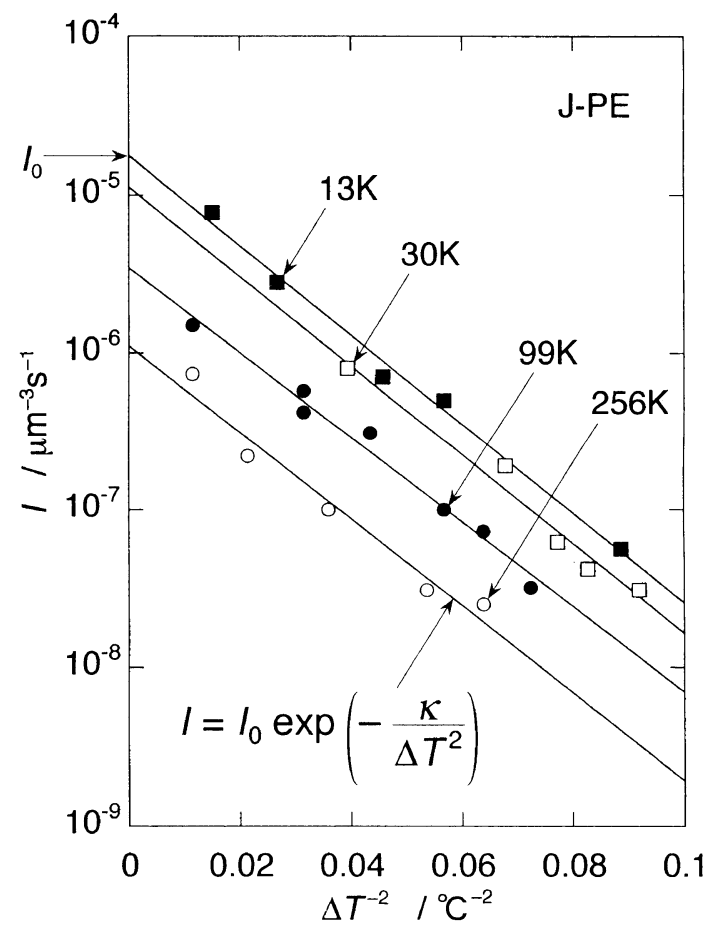

Figure 7. Plot of $\log I$ against $\Delta T^{-2}$ in $M_{n}=13 \mathrm{~K}, 30 \mathrm{~K}, 99 \mathrm{~K}$, and $256 \mathrm{~K}$. The lines show the best fit of the plots, which correspond to the well known formula of the primary nucleation.

the change in $T$ is small enough $(T \simeq 500 \pm 3 \mathrm{~K})$, so the change in $I_{0} \propto \exp \left(-\Delta E^{*} / k T\right) P_{\mathrm{s}}$ can be neglected.

\section{$M_{n}$ Dependence of Nucleation Rate}

What Controls $M_{n}$ Dependence of I? It should be noted that all lines are nearly parallel and shift downwards with increase in $M_{n}$ in Figure 7 . This means that $\kappa$ does not depend on $M_{n}$, while $I_{0}$ decreases with in-

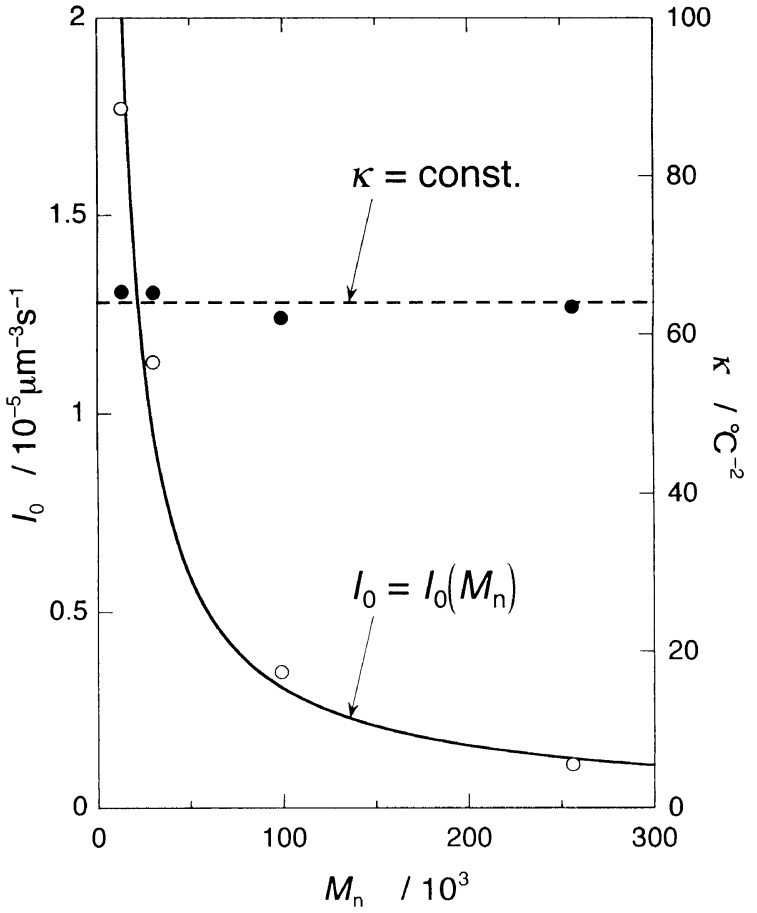

Figure 8. Plot of $I_{0}$ and $\kappa$ against $M_{n}$. The solid line and broken line show the best fit of $I_{0}$ and $\kappa$, respectively. Only the $\kappa$ depends on $M$, while $I_{0}$ does not.

crease in $M_{n} . M_{n}$ dependences of $I_{0}$ and $\kappa$ are quantitatively shown in Figure 8. This clearly shows that

$$
I_{0}=I_{0}\left(M_{n}\right) \text { and } \quad \kappa \simeq \text { const . }
$$

This means that "expanded diffusion constant" $D$ proportional to $I_{0}$ (eq 6) decreases with increase in $M_{n}$, while free energy of forming a critical nucleus $\Delta G^{*}$ related to $\kappa$ (eq 8 and 10 ) does not depend on $M_{n}$. Combination of eq 11 with eq 2 and 6 gives a relation,

$$
I\left(M_{n}\right) \propto I_{0}\left(M_{n}\right) \propto D\left(M_{n}\right)
$$

Thus we have the first conclusion that $M_{n}$ dependence of $I$ is mainly controlled by that of "expanded diffusion process" of polymer chains and not by that of formation process of a critical nucleus.

Power Law of the Primary Nucleaion. Figure 7 shows that $I$ decreases with increase in $M_{n}$ for a constant $\Delta T$. This is quantitatively shown by plots of $\log I$ against $\log M_{n}$ for $\Delta T=3^{\circ} \mathrm{C}, 4^{\circ} \mathrm{C}$, and $9^{\circ} \mathrm{C}$ in Figure 9 . Figure 9 shows that $\log I$ decreases linearly with increase in $\log M_{n}$ for all $\Delta T$ s. It should be noted that all lines showed nearly the same slope $\simeq-1$, which obviously corresponds to the observed fact that $\kappa$ does not depend on $M_{n}$ in Figure 8. Thus we have the following experimental formula,

$$
I\left(M_{n}\right) \propto M_{n}^{-1} \text { for any constant } \Delta T
$$

Therefore $\log I_{0}$ versus $\log M_{n}$ should show the same slope $\simeq-1$, as is confirmed by plots of $\log I_{0}$ against $\log \mathrm{M}_{n}$ in Figure 10. Therefore we have a similar relation that

$$
I_{0}\left(M_{n}\right) \propto M_{n}^{-1}
$$

Combination of eq 12,13 , and 14 gives a universal formula, 


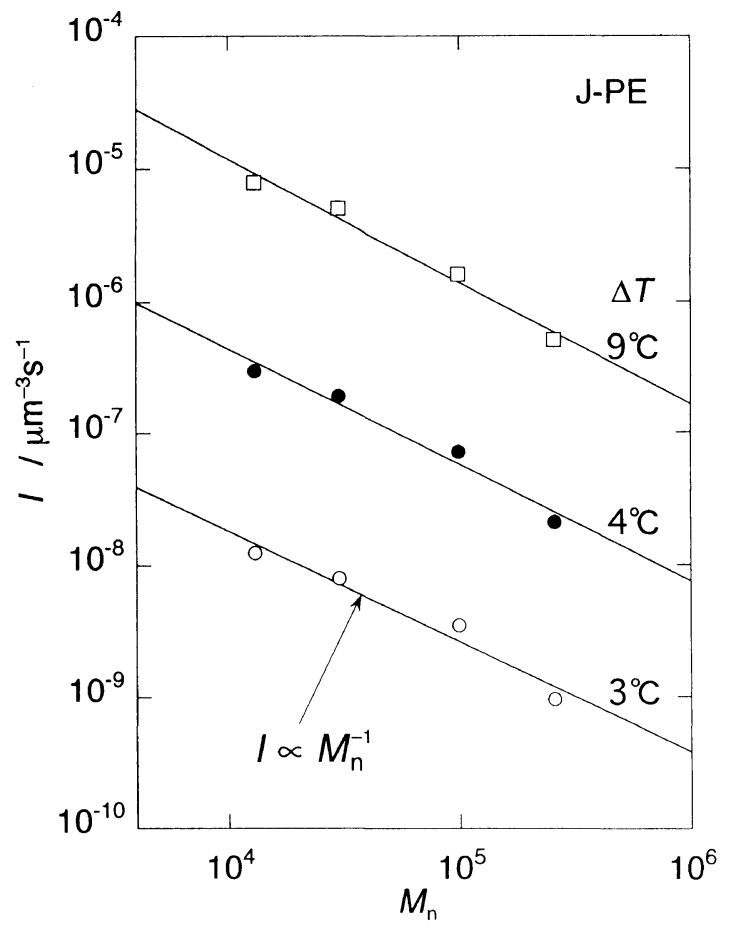

Figure 9. Plot of $\log I$ against $\log M_{n}$ at $\Delta T=3^{\circ} \mathrm{C}, 4{ }^{\circ} \mathrm{C}$, and $9^{\circ} \mathrm{C}$. The lines show the best fit of the plots which correspond to eq 13 .

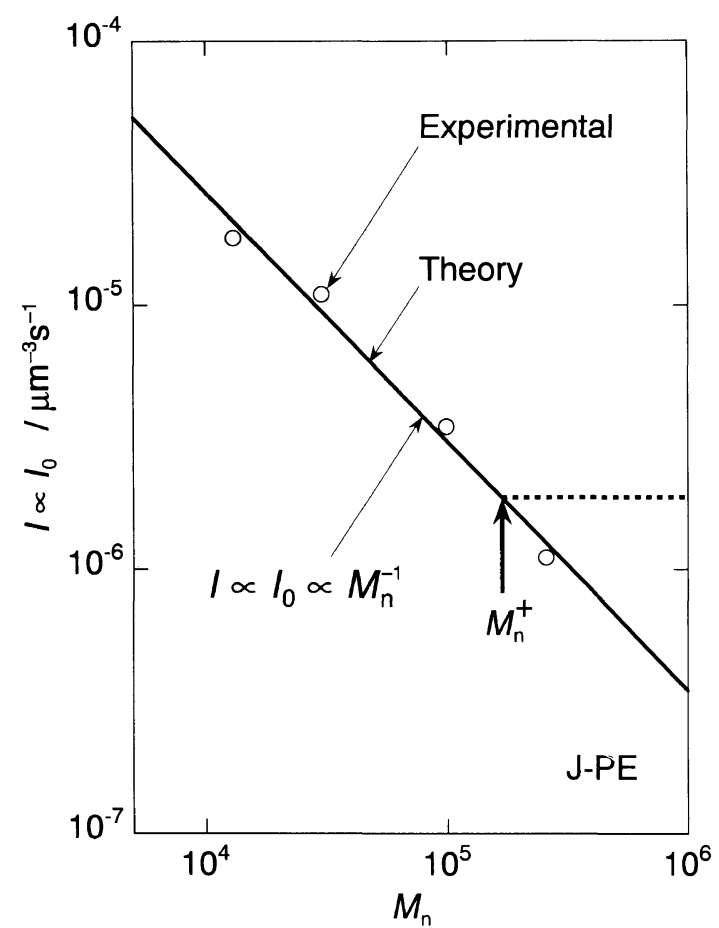

Figure 10. Plot of $\log I_{0}$ against $\log M_{n}$. The lines show the best fit of the plots which correspond to eq 15 indicating the "power law of primary nucleation." The line also corresponds to the theoretical one given by eq 31 and 33 .

$$
I\left(M_{n}\right) \propto I_{0}\left(M_{n}\right) \propto D\left(M_{n}\right) \propto M_{n}^{-1}
$$

Thus we have the second important conclusion that nucleation rate $I$ is in proportion to $M_{n}^{-1}$. We will name this relation the "power law of primary nucleation."

\section{DISCUSSION}

\section{Reason Why $\Delta G^{*}$ Does Not Depend on $M_{n}$}

It was concluded that the $M_{n}$ dependence of $I$ is mainly controlled by that of "expanded diffusion process of chain molecules" and that the formation process of a critical nucleus does not depend on $M_{n}$, that is, $I\left(M_{n}\right) \propto D\left(M_{n}\right)$ and $\Delta G^{*}\left(M_{n}\right)=$ const. The reason of this is due to that the total molecular length included within a critical nucleus $\left(M_{n}^{*}\right)$ in this study is much smaller than molecular length of one chain, which has been shown in the succeeding paper. ${ }^{26}$

$$
M_{n}^{*} \leq M_{n}
$$

This means that only partial length of one chain forms a critical nucleus, while whole length, i.e., $M_{n}$, does not have any important role in the formation of a critical nucleus. Therefore $M_{n}$ does not affect $\Delta G^{*}$.

\section{$M_{n}$ Dependence of $D$ Is Controlled Only by $P_{\mathrm{s}}$}

It was shown that $D \propto \exp \left(-E^{*} / k T\right) P_{\mathrm{s}}$ in eq 6 in introduction and that $D \propto M_{n}^{-1}$ in eq 15 in Result. $\Delta E^{*}$ is $\Delta E$ for the stage of a critical nucleus. It is mentioned in the introduction, $\Delta E^{*}$ is a function of the lamellar thickness of the critical nucleus $\left(l^{*}\right)$. As it has been also shown above that the critical nucleus is composed of a partial length of chains, so $l^{*}$ should be much smaller than the chain length, i.e., $l^{*}$ is not a function of $M_{n}$. Therefore $\Delta E^{*}$ will not depend on $M_{n}$. Therefore present study clearly leads a conclusion that $M_{n}$ dependence of $D$ is mainly controlled by $P_{\mathrm{s}}$. Thus we have a conclusion that

$$
I\left(M_{n}\right) \propto D\left(M_{n}\right) \propto P_{\mathrm{s}}\left(M_{n}\right)
$$

\section{Necessary and Sufficient Condition for Completion of} Nucleation

Equation 17 has important physical meaning as will be discussed below. The nucleation process is schematically illustrated in Figure 11 which was first given in ref 17. This model, we will name "chain sliding diffusion model of nucleation." The nucleation rate $I \propto D \exp \left(-\left(\Delta G^{*} /\right.\right.$ $k T)$ ) given by eq 7 is a product of two factors, $D$ and $\exp \left(-\left(\Delta G^{*} / k T\right)\right)$.

The latter is the probability of the critical nucleation which corresponds to that of reaching at the activation barrier in the Figure 11. The former is an expanded diffusion co-efficient which corresponds to the probability for a nucleus to survive into sufficiently big one after passing through the activation barrier. In another word, the latter is a necessary condition for nucleation, while the former is a sufficient condition.

The maxima of the $\Delta G^{*}$ (positive) corresponds to the thermodynamically unstable point, so the free energy $\Delta G$ can decrease in both growing and melting processes, so the formation of a critical nucleus can not be sufficient condition. The nucleus will be able to survive only when the $\Delta G$ will decrease down to some large amount of negative value. In this case, the forward (i.e., growing) flow will become much more dominant than the backward (i.e., melting) flow. In this case, the $P_{\mathrm{s}}$ becomes significantly large, so the sufficient condition for nucleation is that the $P_{\mathrm{s}}$ becomes large enough. 


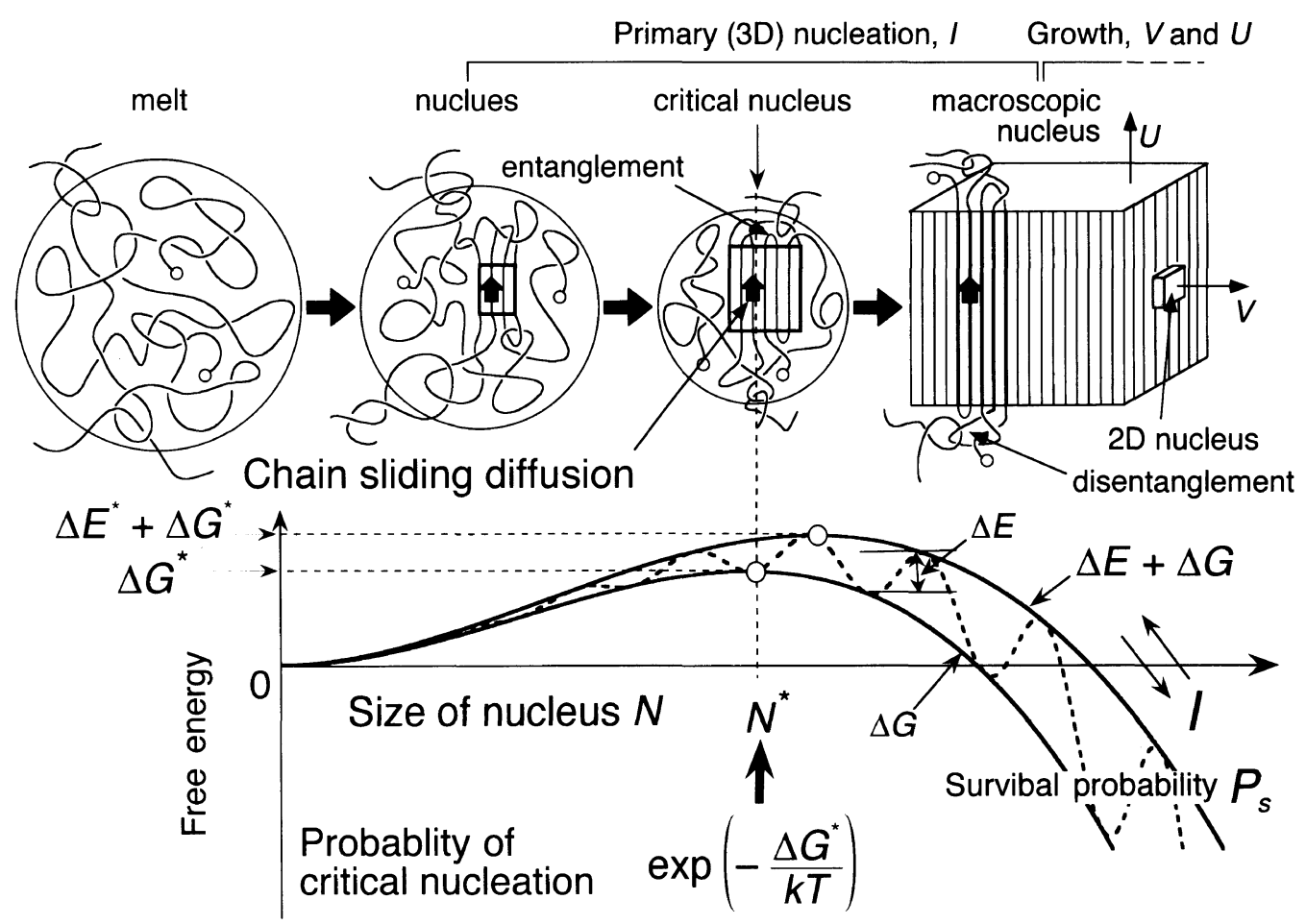

Figure 11. A schematic illustration of proposed "chain sliding diffusion model of primary nucleation". Polymer chains are rearranged from Gaussian shape within the melt into a nucleus through "chain sliding diffusion" within the nucleus and disentanglement within the interface. The bottom indicates change in free energy of the nucleus. See the text.

Therefore the completion of nucleation should be the stage where the $P_{\mathrm{s}}$ becomes large enough. We will denote the critical size of the nucleus which satisfies the sufficient condition $M_{n}^{+}$. Here the size is counted by the molecular weight included within the nucleus.

The combination of above discussion and the obtained conclusion that $M_{n}$ dependence of $D$ is mainly controlled by $P_{\mathrm{s}}$ leads that the critical size of the large nucleus $M_{n}^{+}$depends on $M_{n}$.

$$
M_{n}^{+}=M_{n}^{+}\left(M_{n}\right)
$$

This means that the critically large nucleus should be sensitive to the whole chain length of a chain. This is possible only when the nucleus includes the whole length of a chain (i.e., $M_{n}$ ) within the nucleus. In other words, the $P_{\mathrm{s}}$ can become large enough only when a whole chain is included within a nucleus as is illustrated in Figure 12.

This logic can be confirmed easily as follows. If we assume that the $M_{n}^{+}$does not depend on $M_{n}$, the $I$ will not depend on $M_{n}$ for the polymers with $M_{n} \geq M_{n}^{+}$, so the line of $I(M)$ should show breaking at $M_{n}=M_{n}^{+}$as is illustrated by a broken line in Figure 10 .

As the observed fact is that $I(M)$ does not show any breaking in Figure 10, the critical molecular weight $M_{n}^{+}$ should depend on $M_{n}$, so far as within the range of observed molecular weight. Thus it is confirmed that the above assumption is not correct.

\section{CHAIN SLIDING DIFFUSION THEORY OF NUCLEATION}

The $M_{n}$ dependence of $I$ was expressed by the "power law of primary nucleation," $I \propto M_{n}^{-1}$, in the case of hexagonal phase of PE where ECC is formed. This can be well explained by the following "chain sliding diffusion theory of nucleation."

\section{Chain Sliding Diffusion Model of Nucleation}

The "chain sliding diffusion model of nucleation" is schematically shown in Figure 11. The nucleation process can be divided into three stages; 1) small nuclei (=embryos) are frequently generated and diminished by thermal fluctuation within the melt, 2) size of some of the nuclei will become larger than that of a critical nucleus (=necessary condition for nucleation), and 3 ) finally a little number of nuclei will become sufficiently large ones (named "macroscopic nucleus"), which ensures large survival probability (=sufficient condition for nucleation).

In this theory, we do not care difference between homogeneous and heterogeneous nucleation. Because the difference reflects only in that in $C$ in eq 8 which will be discussed in detail in the succeeding paper ${ }^{26}$ and there is no essential difference in the molecular mechanism of nucleation studied in this study.

It should be stressed in this study that a nucleus can grow into a large one through the "chain sliding diffusion" within the nucleus and "disentanglement" of chains within the interface between the nucleus and the melt. It is obvious that without chain sliding diffusion and disentanglement any nuclei will not be able to grow into large ones. The requirements are due to the topological nature of polymer chains. It is also natural to consider that the chain sliding diffusion and disentanglement become more difficult with increase in $M_{n}$, which should be the origin of $M_{n}$ dependence of $I$. 


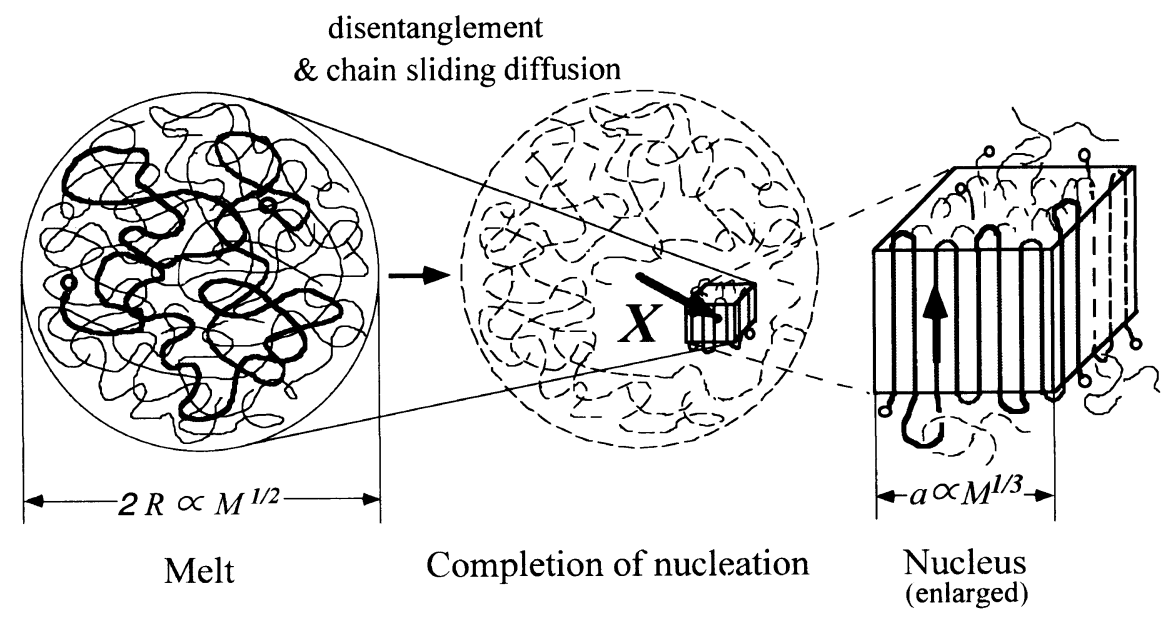

Figure 12. A schematic illustration of conformational change of a chain in nucleation process from Gaussian spherical one within the melt into folded one within a nucleus. The completion of nucleation corresponds to the stage where a whole chain is involved within a nucleus. A nucleus includes a few or several chain molecules. $X$ is displacement of the center of mass during nucleation.

\section{Nuclei Will Be Formed within a Gaussian Sphere}

It is well known that a chain takes Gaussian conformation within the melt and that a lot of chains are superimposed within the same space where the Gaussian sphere occupies, as is illusrated in Figure 12. It is also well known that the radius of the Gaussian sphere $(R)$ and the number of superimposed chains within the sphere are both in proportinal to $\sqrt{M_{n}}$ for chains with $M_{n}$,

$$
R \propto \sqrt{M_{n}}
$$

so a lot of number of chains are usually superimposed in the case of polymers with usual molecular weight.

At the stage of completion of nucleation, a whole chain should be involved within a nucleus, as has been concluded in the previous section. It is natural to consider that the nucleus is a type of folded chain one, because it is well known that bundle-like nucleus is unusual in the case of nucleation from the melt ${ }^{10}$ So most adjacent stems within the nucleus are connected by folds. In another word, most stems are folded from the same chain. In this case, the nucleus at the above stage should be composed of a few or several or at most ten chains. Here only order of the number of chains within a nucleus is discussed and it is obvious that a nucleus cannot include all number of chains which were included within Gaussian sphere. Therefore the lateral dimension of the nucleus $(a)$ can be roughly given by

$$
a \propto M_{n}^{1 / 3}
$$

Thus superimposed chains within the melt should be separated (segregated) with each other into different nuclei in the nucleation process, as illusrated in Figure 12. In another word, an expanded chain within a sphere converges into a smaller volume of nucleus. Of course, this does not mean that all chains will be nucleated at all. Under low degree of supercooling as this study, nucleation rate is not large, so it is usual that only a little nucleus will be formed within the Gaussian sphere. After the primary nucleation, sample will be solidified through succeeding growth process.

Therefore a little number of nuclei will be statistically formed within the Gaussian sphere. It is natural to assume that the position of the generated nucleus will be statistically scattered within the sphere. So in the nucleation process, the center of mass should move from the center of the Gaussian sphere to some other place within the sphere. The displacement of which is denoted as $X$ (Figure 12).

The density-distribution function of a nuclei as a function of $X$ within the sphere, denoted by $f(X)$, can be given by

$$
f(X) \mathrm{d} X=4 \pi X^{2} \mathrm{~d} X
$$

The average of $X,\langle x\rangle$, is then approximately given by

$$
\langle X\rangle=\frac{\int_{0}^{R} X f(X) \mathrm{d} X}{\int_{0}^{R} f(X) \mathrm{d} X}
$$

Insertion of eq 21 into eq 22 leads

$$
\langle X\rangle=R \propto \sqrt{M_{n}}
$$

where eq 19 is combined.

\section{Formulation of Molecular Weight Dependence of $D$}

It is the essential point in this theory that the displacement $X$ corresponds to the expanded diffusion coefficient $D$, because the diffusion is directly required for completion of nucleation. The expanded $D$ corresponds to the conformational change of a chain from Gaussian one into folded one within a nucleus, which requires the disentanglement and chain sliding diffusion within a nucleus.

Here we may assume that the $D$ can be formulated by well known classical diffusion theory, because the expanded diffusion process is a kind of diffusion one even though it reflects topological nature of polymer chains such as disentanglement, so the phenomenological classical diffusion theory should be applicable. We will describe the $D$ using well known Fick's law,

$$
D=\langle X\rangle^{2} / \tau
$$

where $\tau$ is relaxation time for the diffusion.

The diffusion process can be also regarded as chain 
sliding diffusion one necessary for the completion of nucleation. The sliding-diffusion coefficient $\left(D_{\text {slid }}\right)$ can be similarly given using Ficks law,

$$
D_{\text {slid }}=x^{2} / \tau
$$

where $x$ is the total displacement measured along chain axis necessary for the sliding diffusion in completion of nucleation.

The $D_{\text {slid }}$ can be also expressed using well known Einstein's relation,

$$
D_{\text {slid }}=k T / \zeta
$$

where $\zeta$ is effective friction coefficient for the sliding diffusion mentioned above.

Combination of eq 24, 25, and 26 gives the basic formula of $D$,

$$
D=\frac{k T}{\zeta} \frac{\langle X\rangle^{2}}{x^{2}}
$$

The next problem is to formulate the $M_{n}$ dependence of $x$ and $\zeta$. It is obvious that $\zeta$ is in proportion to the $x$, because the friction should be in proportion to the total distance of displacement,

$$
\zeta \propto x
$$

$x$ is total displacement in sliding diffusion in completion of nucleation, i.e., displacement necessary for rearrangement of a chain from Gaussian conformation into folded conformation within a nucleus. So it is obvious that $x$ should inrease with increase of $M_{n}$. The rearrangement should be easy in the case of the mobile crystalline phase, because chain can slide easily within the nucleus or in the case that interchain friction is less due to molecular shape which basically depends on species of materials. Therefore $x$ is an increasing function of $M_{n}$ and of a kind of order parameter $(\alpha)$ which is related to the degree of order of the crystalline phase or species of materials. On the basis of this consideration, we will assume without any definite basis at this stage that

$$
x \propto M_{n}^{\alpha}
$$

Insertion of eq 23,28 , and 29 into 27 leads the formula of $D$,

$$
D\left(M_{n}\right) \propto M_{n}^{1-3 \alpha}
$$

Formulation of Molecular Weight Dependence of I

Combination of eq 17 and 30 gives the final formua of $I\left(M_{n}\right)$,

$$
I\left(M_{n}\right) \propto D\left(M_{n}\right) \propto M_{n}^{1-3 \alpha}
$$

This final formula of $I\left(M_{n}\right)$ is a kind of power law, so the observed "power law of nucleation rate" given by eq 15 can be well explained by the chain sliding diffusion theory of nucleation.

\section{Comparison between Theory and Experiment}

Comparison between observed power law, $I \propto D \propto$ $M_{n}^{-H}$ where $H$ is a constant, and theoretical one, $I \propto D \propto M_{n}^{-3 \alpha}$ gives the relation between power $H$ and order parameter $\alpha$

$$
H=3 \alpha-1
$$

Thus it is shown that the power $H$ is directly related to order parameter of crystallin phase. As $H=1$ in this work, we have,

$$
\alpha=2 / 3
$$

The further study on the dependence of $\alpha$ on the crystalline order or species of materials should be interesting to confirm the theory.

\section{CONCLUSION}

1. We have a new experimental formula of $M$ dependence of $I$, that is, $I(M) \propto M^{-1}$, which we named "power law of primary nucleation" in polymer system.

2. It was concluded that $M$ dependence of $I$ is mainly controlled by the "chain sliding diffusion" process not by the formation process of a critical nucleus, that is, $I(M) \propto D(M) \propto P_{\mathrm{s}}(M)$ and $\Delta G^{*}=$ const. Here $D$ is an "expanded diffusion constant" expanded to topological linear chain systems, $P_{\mathrm{s}}$ is a survival probability in "chain sliding diffusion" theory and $\Delta G^{*}$ is activation free energy forming a critical nucleus.

3. It is concluded that primary nucleation is a process of "chain sliding diffusion" within the nucleus which requires disentanglement of molecular chain within the interface between the nucleus and the melt.

4. Chain sliding diffusion theory of nucleation was presented. The theory leads the power law of $I\left(M_{n}\right) \propto$ $D\left(M_{n}\right) \propto M_{n}^{1-3 \alpha}$ where $\alpha$ is a constant. This well explained the observed power law.

Acknowledgments. The authors are grateful to the financial supports by International Joint Research grant, NEDO, 1995-1998 and Grant-in-Aid for Scientific Research (B) (No. 09450364) and Professor K. Iwata of Fukui University for helpful discussions.

\section{REFERENCES}

1. A. Ookawa, in "Kessyo-Seicho (Crystal Growth)," Shokabo, Tokyo, 1977, Chapter 1.

2. D. Turnbull and J. C. Fisher, J. Chem. Phys., 17, 71 (1949).

3. D. Turnbull, J. Chem. Phys., 18, 198 (1950).

4. A. G. Walton, in "Nucleation," Marcel Dekker, Inc., New York, N.Y., 1969, Chapter 5.

5. W. A. Tiller, "The Science of Crystallization," Cambridge University Press, New York, N.Y., 1991, Chapter 8.

6. J. I. Lauritzen and J. D. Hoffman, J. Res. NBS, 64A, 73 (1960).

7. A. Sharples, Polymer, 3, 433 (1962).

8. F. J. Limbert and E. Bear, J. Polym. Sci., 1A, 3317 (1963).

9. J. N. Hay, J. Polym. Sci., 3, 433 (1965).

10. F. P. Price, in "Nucleation," Marcel Dekker, Inc., New York, N.Y., 1969, Chapter 8.

11. M. Nishi, M. Hikosaka, A. Toda, and M. Takahashi, Polymer, 39, 1591, (1998).

12. M. Okada, M. Nishi, M. Takahashi, H. Matsuda, A. Toda, and M. Hikosaka, Polymer, 39, 4535 (1998).

13. M. Hikosaka, in "Advances in the Understanding of Crystal Growth Mechanism," Elsevier Sci. Ltd., Amsterdam, 1996, Chapter 3.

14. P. G. De Gennes, J. Chem. Phys., 55, 572 (1971).

15. M. Doi and S. F. Edwards, in "The Theory of Polymer Dynamics," Oxford University Press Inc., New York, N.Y., 1986.

16. R. Becker, and W. Döring, Ann. Phys., 24, 719 (1935).

17. M. Hikosaka, Polymer, 28, 1257 (1987).

18. M. Hikosaka, Polymer, 31, 458 (1990).

19. A. Keller, Phil. Mag., 2, 1171 (1957). 


\section{NisHi et al.}

20. B. Wuderlich and T. Arakawa, J. Polvm. Sci., 2A, 3697 (1964).

21. F. C. Frank and M. Tosi, Proc. R. Soc. London, 263A, 323 (1961).

22. B. Wunderlich and G. Czornyj, Macromolecules, 10,906 (1977).

23. M. Hikosaka, S. Rastogi, A. Keller, and H. Kawabata. J. Macromol. Sci. Phys., B31, 87 (1992).
24. M. Hikosaka, K. Tsukijima, S. Rastogi, and A. Keller, Polymer, 33, 2502 (1992).

25. M. Hikosaka and S. Tamaki, J. Phys. Soc. Jpn., 50, 638 (1981).

26. M. Hikosaka, M. Nishi, and S. K. Ghosh, submitted to Polym. $J$. 